\title{
HOW DO CHANGES IN THE EXTERNAL ENVIRONMENT AFFECT DRIVING ENGAGEMENT IN AUTOMATED DRIVING? - AN EXPLORATORY STUDY
}

\author{
Heishiro Toyoda, Josh Domeyer, John Lenneman \\ Toyota Technical Center, Toyota Motor North America, Ann Arbor, MI, USA \\ Email: heishiro.toyoda@toyota.com,joshua.domeyer@toyota.com,john.lenneman@toyota.com
}

Summary: Driving is performed while processing various internal driver and external cues from the driving environment (e.g., subtle vibrations, lateral and longitudinal acceleration). The present study was conducted for the purpose of identifying how much external cues affect driver's gaze behavior in an automated driving environment. Fifteen participants drove a commercially available vehicle with longitudinal and lateral automation on an oval test track. Participants were asked to drive the vehicle with and without automation, with or without a side-task, and either with their hands-on or hands-off-wheel. Driver's gaze behavior, handson-wheel status and driving conditions were annotated from video data. The results showed that during automated driving and side-task performance, eyes-on-road time was significantly greater after entering a curve than before and as a result of changes in speed. These differences were not observed in automated driving mode when no side-task is performed. Also, these were more sensitive than hands-on or hands-off-wheel conditions. The results also suggest that drivers may process nonvisual information (e.g., vestibular information produced by changes in lateral and longitudinal vehicle acceleration) prior to or even during the implementation of a visual resource allocation strategy. The present study suggests driver awareness can be aided without requiring the driver to grab the steering wheel.

\section{INTRODUCTION}

One of the big challenges automotive engineers face when designing automated vehicles is how to achieve a good balance between driver comfort and safe operations when there are system limitations. The Society of Automotive Engineers (SAE) defined vehicle automation levels with six different levels (SAE J3016, 2016). A level 2 automated vehicle requires the driver to complete object and event detection and response (OEDR) as a subtask of the dynamic driving task while the system performs sustained lateral and longitudinal vehicle motion control. On the other hand, a level 3 automated vehicle does not need the driver to perform OEDR but does require the driver to be receptive to a request to intervene and respond by performing a fallback action. If the driver has enough trust in the system, the driver may operate the vehicle in a manner such that a vehicle defined as level 2 can become situationally level 3 . As a result, the driver may engage in non-driving related tasks and may perform an insufficient amount of OEDR behavior. The lack of enough OEDR is likely to create vulnerabilities in transfer of control when the system confronts limitations. To address this issue while also avoiding driver annoyance, development of human machine interfaces (HMIs) that induce an increase in OEDR behavior should be explored.

Considering that driving likely involves the processing of various external cues beyond visual and auditory (e.g., subtle vibrations, lateral and longitudinal acceleration, road noise), one strategy in HMI design for automated vehicles could include the enhancement of additional 
sensory information. However, prior to development, it is important to gain a deeper understanding of what and how drivers process sensory information while driving an automated vehicle. Therefore, we have designed the present study to answer questions regarding what driving cues can be effective for maintaining or controlling driver awareness in a situationally level 3 automation condition, and whether this effect varies as a function of automation level.

The present study investigated the effects of external driving cues on driver awareness as measured by driver visual behavior. Participants drove a closed course test track with hands-on or hands-off-wheel and while occasionally engaging in a side-task. An analysis of gaze behavior during changes in speed and during different road configurations (e.g., straight and curve) were included so as to compare the effects of external driving cues on driver awareness. Hands-on and hands-off the steering wheel were analyzed to compare their effect on driver awareness. Finally, the impact of a side-task on the driver's eye gaze behavior in automated driving was also investigated.

\section{METHOD}

\section{Participants}

Fifteen automotive industry employees (e.g., engineers, market researchers) participated in the study (Age: $M=32, S D=8.7$, Female = 3). Informed consent was obtained from the participants before the experimental drive. All participants were naïve users of vehicle automation and, thus, had little to no experience using vehicle automation.

\section{Apparatus and Measures}

A commercially available vehicle with longitudinal and lateral automation features that enable situationally level 3 automated driving was selected to use in the study. For this study a situationally level 3 vehicle is one that could drive around the closed course without a requirement to continuously monitor the roadway (e.g., OEDR). No changes on the original system (e.g., sensors and warning systems) were made. The system allowed for two minutes of hands-off-wheel driving after which a visual and audible warning was provided to indicate the need for the driver to apply torque to the steering wheel. The side-task was presented on an additional display (Microsoft Surface Pro, 12.3 in) which was placed in front of the native center stack display for interacting with a side-task.

Two video cameras (1280 x 720 pixels, 30fps) were instrumented in the vehicle: one was pointed toward the driver's face while the other faced the instrument panel and forward roadway. Eye gaze data were manually coded from the video data by a single coder using the ISO 15007 standard (2014). Eye gaze data were categorized to three types; forward roadways (eyes forward, or eyes-on-road), the instrument panel (eyes-on-meter), and other (eyes-on-other) including looking at a display presenting the side-task. Driving environment, visual warning status and vehicle speed were also annotated from the video data. 


\section{Procedure and Tasks}

The oval course was located at the FT Techno of America Fowlerville Proving Grounds. It is 4.8

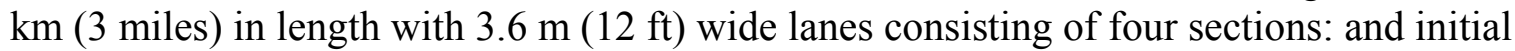
straightaway $1.3 \mathrm{~km}$ long (4,300 ft), a curve (herein referred to as $\mathrm{R} 1500)$ with a radius that begins at $457 \mathrm{~m}(1500 \mathrm{ft})$ and decreases to $305 \mathrm{~m}(1000 \mathrm{ft})$, a second straightaway $1.4 \mathrm{~km}$ long $(4,500 \mathrm{ft})$, and a second curve (herein referred to as R1000) with a constant radius of $305 \mathrm{~m}$ $(1000 \mathrm{ft})$. Participants drove 8 laps, each lap taking approximately 4 minutes and the total session lasting about $1 / 2$ hour. The laps included a mix of manual driving baselines, automated driving baselines without a side-task, and automated driving with a side-task (Table 1). Participants drove in a single lane on the closed course and did not execute additional maneuvers (e.g., lane change, stopping). Automated driving with a side-task was split into two conditions presented sequentially and counterbalanced across participants: two laps with hands-on-wheel and 2 laps with hands-off-wheel (four automated driving laps with side-task in total).

Participants were asked to follow a lead vehicle driven by a trained driver at a constant speed of $72.4 \mathrm{~km} / \mathrm{h}$ (45 mph). However, on the first straightaway the trained driver used the steering wheel controls to increase speed by $8 \mathrm{~km} / \mathrm{h}(5 \mathrm{mph})$, which was followed by a decrease in speed by $8 \mathrm{~km} / \mathrm{h}(5 \mathrm{mph})$. These speed change events (which occurred on every lap) were created to study the effect of longitudinal vestibular cues on gaze behavior.

During laps 3-6, participants were asked to perform an email sorting task on the tablet display (Gibson et al., 2016). The task was designed to represent of realistic side-task drivers may try to perform while the vehicle is automated driving mode. Specifically, participants were instructed to sort emails presented in a list into one of three common categories (work, family $\&$ friends, and trash). Participants were allowed to sort emails at their own pace.

Table 1. Experimental conditions

\begin{tabular}{|c|c|c|c|c|}
\hline Lap & Conditions & Automation & Side-Task & $\begin{array}{l}\text { Hands-On- } \\
\text { Wheel }\end{array}$ \\
\hline 0 & Familiarization & - & - & - \\
\hline 1 & Manual Driving Baseline & OFF & $\mathrm{N}$ & $\mathrm{ON}$ \\
\hline 2 & $1^{\text {st }}$ Automated Driving Baseline & ON & $\mathrm{N}$ & Driver's Choice \\
\hline $3,4(5,6)$ & $\begin{array}{l}\text { Automated Driving with Side-Task } \\
\text { with Hand(s)-on-wheel (ASTHon) }\end{array}$ & $\mathrm{ON}$ & $\mathrm{Y}$ & $\mathrm{ON}$ \\
\hline$(3,4) 5,6$ & $\begin{array}{l}\text { Automated Driving with Side-Task } \\
\text { with Hands-off-wheel (ASTHoff) }\end{array}$ & $\mathrm{ON}$ & $\mathrm{Y}$ & OFF \\
\hline 7 & $2^{\text {nd }}$ Automated Driving Baseline & $\mathrm{ON}$ & $\mathrm{N}$ & Driver's Choice \\
\hline
\end{tabular}

\section{RESULTS}

Multiple Wilcoxon signed rank tests were conducted for the eye gaze data. From the annotated data the total duration for each eye gaze category was calculated and converted to a percent for each event. In order to make comparisons of effects caused by various vestibular stimuli from external driving cues, speed change events and curve events were created. For longitudinal vestibular cues, we compared the first straightway (where the previously described changes in speed occurred) to the second straightway (where speed was a constant $72.4 \mathrm{~km} / \mathrm{h}$ ). For lateral vestibular cues, we compared the $15 \mathrm{~s}$ prior to entering the curve to the $15 \mathrm{~s}$ after entering the 
curve for each curve. Further, since one curve had a larger radius (curve R1500) whereas the other curve had smaller radius (curve R1000), the effect of differences in magnitude of lateral vestibular cues on eye gaze can be assessed. Because of the limitations with the study (noted in the discussion), we felt that an assessment of trends in behavior was worthy of exploration as they could lay a foundation for future research.

The effect of speed change is shown in Figure 1. In ASTHoff percent of eyes forward was greater when speed changed on the straightaway compared to when it $\operatorname{did} \operatorname{not}(Z=2.95, p=$ 0.003). The trend was the same for ASTHon $(Z=2.16, p=0.031)$. On the other hand, in the first and second automated driving baselines, no statistically significant differences nor trends in gaze behavior were observed between when speed changed on the straightaway $(Z=0.284, p=0.776)$ compared to when it did not $(Z=0.973, p=0.331)$.

The effect of the curves is shown in Figure 2. The results show a trend that in the automated driving with a side-task and with hands-on-wheel (ASTHon) the curve events evoked an increase in percent eyes forward after entering the curve compared to the $15 \mathrm{~s}$ prior to entering the curve, $(Z=1.93, p=0.054)$, while the trend was not seen in $\operatorname{ASTHoff}(Z=0.398, p=0.691)$.

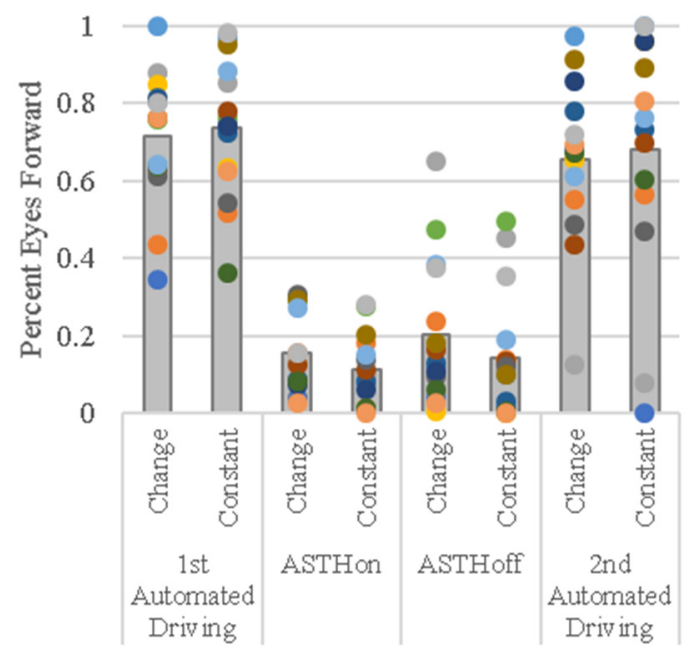

Figure 1. Percent Eyes Forward for Speed Change Events. - the columns show means and the dots show participants data. ASTHon and ASTHoff stand for Automated Driving with SideTask with Hand(s)-on and Hands-off-wheel, respectively.

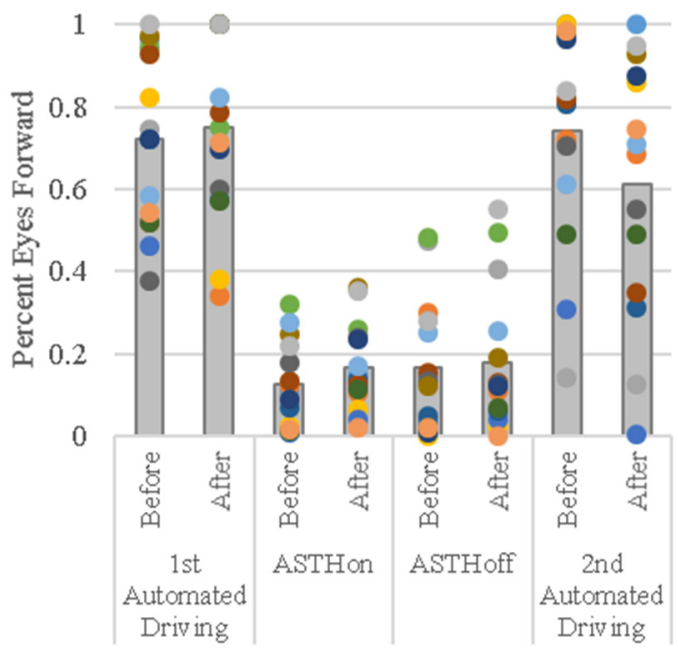

Figure 2.Percent Eyes Forward for Curve Events. - the columns show means and the dots show participants data. ASTHon and ASTHoff stand for Automated Driving with Side-Task with Hand(s)-on and Hands-off-wheel, respectively.

In addition, as shown in Figure 3, when looking deeper at hands-on-wheel alone (ASTHon), there was a significant increase in percent eyes forward for curve R1000 after entering the curve compared to the $15 \mathrm{~s}$ prior to entering the curve $(Z=2.73, p=0.006)$, while there was no significant difference for curve $\operatorname{R} 1500(Z=0.625, p=0.532)$. Interestingly, when looking deeper at hands-off-wheel alone, the trend for curve R1000 was an increase in percent eyes forward $(Z=$ $2.61, p=0.009)$. However, the trend for curve $\mathrm{R} 1500$ was a decrease in percent eyes forward $(Z$ $=2.01, p=0.044)$. 


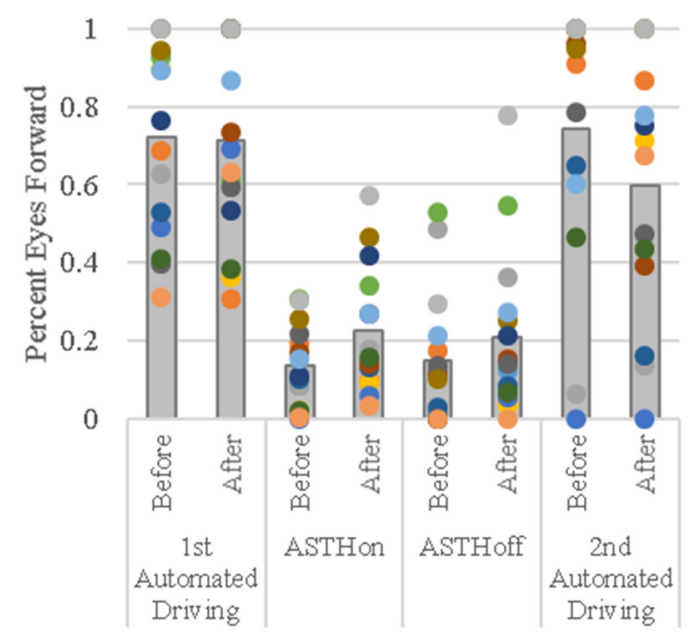

(a) Curve R1000 Event

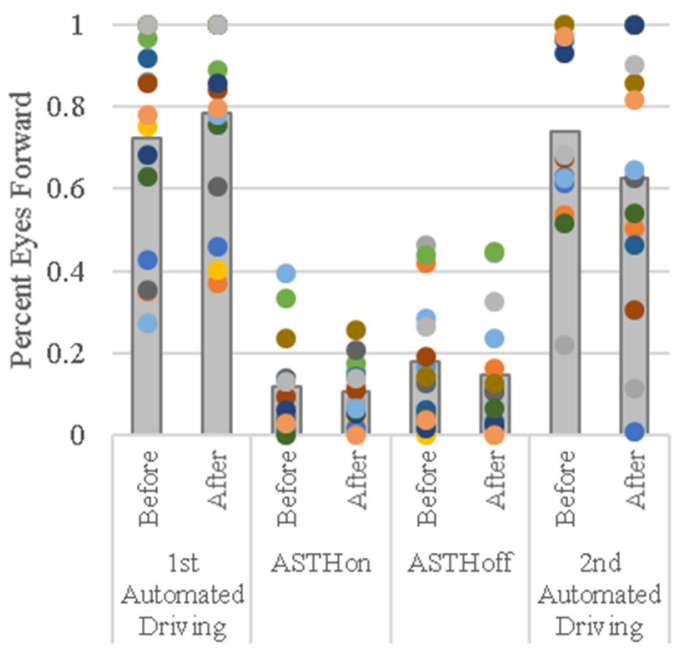

(b) Curve R1500 Event

Figure 3. Percent Eyes Forward by different curve events - the columns show means and the dots show participants data. ASTHon and ASTHoff stand for Automated Driving with Side-Task with Hand(s)-on and Hands-off-wheel, respectively.

Lastly, there was no difference in percent eyes forward between ASTHon and ASThoff conditions $(Z=0.511, p=0.609)$. Further, the results in Figure 4 show the percent of eyes forward decreased significantly from manual driving baseline to both the first automated driving baseline, $(Z=3.07, p=0.002)$ and the second automated driving baseline $(Z=3.35, p<0.001)$. Finally, there was a significant decrease in the percent of eyes forward from the $1^{\text {st }}$ automated driving baseline to $\operatorname{ASTHon}(Z=3.41, p<0.001)$ as well as ASTHoff $(Z=3.41, p<0.001)$.

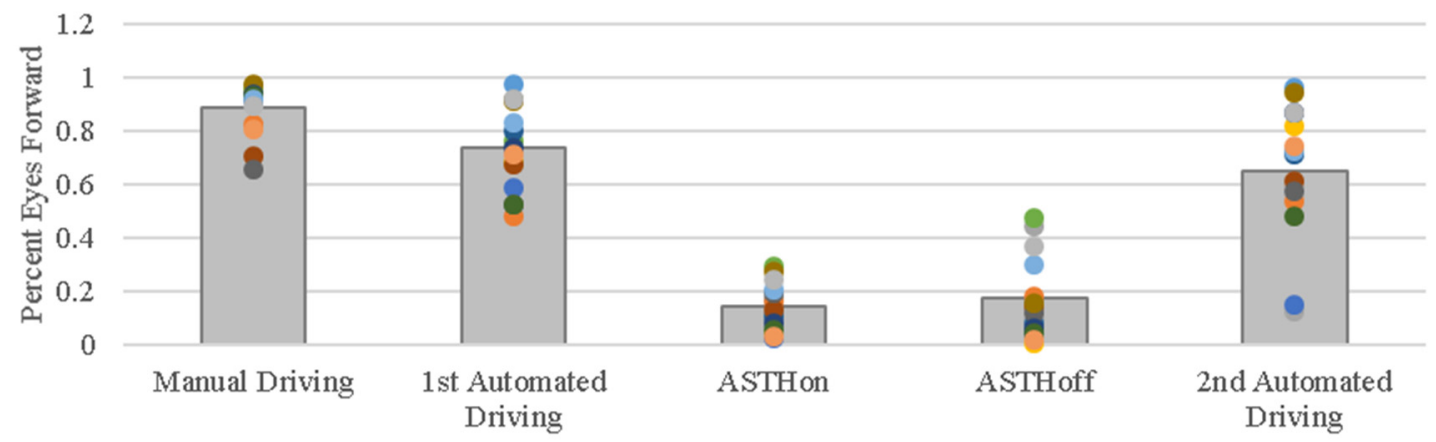

Figure 4. Percent Eyes Forward for Each Trial - the columns show means and the dots show participants data. ASTHon and ASTHoff stand for Automated Driving with Side-Task with Hand(s)-on and Hands-offwheel, respectively.

\section{DISCUSSION}

The objectives of the study were to determine whether eye gaze patterns are affected by handson-wheel or hands-off-wheel, and whether eye gaze patterns are affected by external driving characteristics such as speed changes and curves. As Figure 4 shows, there were no differences in gaze behavior when comparing hands-on and hands-off-wheel in automated driving while performing a side-task. This is consistent with recent research demonstrating that maintaining 
hands-on-wheel and foot-on-pedal did not improve reaction time to emergency events (Ikeda et al., 2016). On the other hand, the differences in speed change (Figure 1) and curve events (Figure 2) showed meaningful trends. An increase in eyes forward during a speed change or entering a curve could be an indication that vestibular cues may be part of a larger visual attention allocation strategy that when implemented should help the driver maintain awareness.

Interestingly, the results in Figure 3 show trends in differences between different curve events, where entering a smaller radius curve increased percent eyes forward. The difference in gaze patterns between the two curves could be due to the smaller radius curve (radius $305 \mathrm{~m}$, curve R1000) evoking relatively higher lateral acceleration compared to the other curve (radius $457 \mathrm{~m}$, curve R1500), and this lateral acceleration may induce higher percentage of gazing forward to the roadway compared to the percentage just before entering the curve. This is consistent with research conducted by Kishi, Akamatsu, and Endo (2016) in which they demonstrated eyes-onroad time increased while driving in a smaller radius curve. However, they conducted their research in a driving simulator which was absent of vestibular cues. Thus, while collectively Kishi et al. (2016) and this study suggest differences in gaze behavior may exist between the curves of varying radii, further research would need to be conducted to delineate the contributions of vestibular and visual flow cues on gaze behavior.

Finally, the difference in gaze behavior between curve R1000 and R1500 may be explained with the task-capability interface model by Fuller (2005). The task-capability model suggests that a driver makes a strategic decision (based on task demands including vehicle speed, road shape and the number of road users) regarding attention and effort allocation to driving. When applied to the tasks in the present study, predicted task demands of curve R1000 (e.g., increased precision/control needed to avoid road departures) may impact the percent eyes forward to the road.

\section{Limitations}

First, while the tasks were performed on a test track with a real production vehicle, there is still an artificial component to the experiment and it is acknowledged that participants' behaviors might be different from real-world situations. The subtle cues that we tried to isolate and investigate in this study might be a small portion of a larger set of informational cues (vestibular combined with haptic, visual, etc.) that can be processed in a more naturalistic driving environment. Second, while visual inspection of video data did not capture obvious compensatory behaviors, future analyses should include side-task performance data to provide insight into task prioritization. Finally, insight into strategies used during task performance might also benefit from an analysis of driver hand placement on the wheel during driving baselines.

\section{CONCLUSION}

Two significant observations were pulled out as a result of this study. First, when the driver continuously performs a side-task which requires eyesight to be away from the forward roadway, requiring a hands-on-wheel may not ensure that drivers also keep their eyes-on-road. Second, the vestibular cues as a result of changes in longitudinal and lateral acceleration can be used to help promote eyes-on-road and help the driver get back to monitoring the forward roadway to 
some extent. With regard to potential applications, the present study suggests that innovating a system that can support driver comfort and situational improvement of driver awareness (through the improvement of vestibular cues) at the same time might be worth exploring.

\section{REFERENCES}

Fuller, R. (2005). "Towards a general theory of driver behavior". Accident Analysis and Prevention Vol. 37, 461-472.

Gibson, M., Lee, J., Venkatraman, V., Price, M., Lewis, J., Montgomery, O., \& Mutlu, B. (2016). "Situation Awareness, Scenarios, and Secondary Tasks: Measuring Driver Performance and Safety Margins in Highly Automated Vehicles". SAE Int. J. Passeng. Cars Electron. Electr. Syst., 2016-01-0145.

Ikeda, T., Utsumi, A., Kawanishi, N., Nagasawa, I., Shiokawa, T., Uchikata, K., \& Hagita, N. (2016). Reaction to Critical Situations druign Automated Driving in Different Driving Positions. JSAE Congress Proceedings (Autumn), 478-482.

ISO 15007. (2014). Road vehicles -- Measurement of driver visual behaviour with respect to transport information and control systems -- Part 1: Definitions and parameters. Switzaland: ISO.

Kishi, H., Akamatsu, M., \& Endo, T. (2016). "Consideration of driving readiness during automated driving - Time prediction to regain driving control, using driver's visual behavior metric and task demand -". JSAE Congress Proceedings (Autumn), 472-477 (20166090).

Price, M., Venkatraman, V., Gibson, M., Lee, J., \& Mutlu, B. (2016). "Psychophysics of Trust in Vehicle Control Algorithms". SAE Int. J. Passeng. Cars - Electron. Electr. Syst. 9(1), 201601-0144.

SAE J3016. (2016). "Surface Vehicle Recommended Practice: Taxonomy and Definitions for Terms Related to Driving Automation Systems for On-Road Motor Vehicles". Warrendale, PA: SAE International. 